\title{
AGRICULTURE GENOMICS
}

\section{Genome structural variation for 100 tomatoes}

Cell https://doi.org/gg23pb (2020)

Understanding the genetic variation that underpins phenotypic variation in crop plants is necessary for designing and engineering favoured alleles for genome editing. Genome structural variations (SVs), which include large deletions, insertions, duplications and chromosomal rearrangements, affect crop improvement and domestication traits such as flowering time, fruit size and stress resistance. Until recently, however, due to the limitations of sequencing technologies, identification of SVs has been difficult and the extent, diversity and quantitative impact of SVs in many crops remain largely unknown.

Michael Schatz from Johns Hopkins University, Zachary Lippman from Cold Spring Harbor Laboratory and their colleagues used the recently developed long-read nanopore sequencing technology to identify $238,490 \mathrm{SV}$ s in 100 diverse tomato accessions, and they generated 14 tomato reference genomes. The group demonstrated that breeding and introgression have shaped SV content, and that multiple SVs modified fruit flavour, size and production. In combination with the genome editing technology CRISPR, they revealed complex genetic interactions among four SVs affecting an important harvesting trait, 'jointlessness' (which allows complete separation of fruits from other floral parts), and outlined the path towards jointless breeding in modern tomato cultivars.

The findings highlight the underexplored role of SVs in genotype-phenotype relationships. Raising the curtain on SVs can facilitate the exploitation of natural and engineered SVs for crop improvement.

Yufang Guo

Published online: 16 July 2020

https://doi.org/10.1038/s43016-020-0125-7 